\title{
Variação espaço-temporal da ictiofauna da zona de arrebentação da Praia do Cassino, Rio Grande do Sul, Brasil
}

\author{
Mauro Sergio Pinheiro Lima ${ }^{1,3} \&$ João Paes Vieira ${ }^{2}$ \\ ${ }^{1}$ Laboratório do Oceano, Departamento de Botânica, Ecologia e Zoologia, Universidade Federal do Rio Grande do Norte. \\ Campus Universitário Lagoa Nova, Caixa Postal 1524, 59072-970 Natal, Rio Grande do Norte, Brasil. \\ E-mail: mauspl@yahoo.com.br \\ ${ }^{2}$ Laboratório de Ictiologia, Departamento de Oceanografia, Instituto de Oceanografia, Fundação Universidade do Rio \\ Grande. Avenida Itália km 8, Caixa Postal 474, 96201-900 Rio Grande, Rio Grande do Sul, Brasil. \\ E-mail: jvieira@mikrus.com \\ ${ }^{3}$ Corresponding author.
}

\begin{abstract}
Spatiotemporal variation in the fish fauna of Praia do Cassino, Rio Grande do Sul, Brazil. This study analyzes the spatiotemporal variation of the ichthyofauna of the surf zone of Praia do Cassino, municipality of Rio Grande (state of Rio Grande do Sul, Brazil) from October 2005 to September 2006. Fishes were collected monthly and during daytime using a large beach seine net $(30 \mathrm{~m} \times 1.8 \mathrm{~m}$, with $12 \mathrm{~mm}$ uniform mesh manufactured with monofilament nylon wire). Five hauls were conducted on each of three sampling sites: one near the jetties of Patos Lagoon and two located $5 \mathrm{~km}$ and $10 \mathrm{~km}$ south from the jetties, respectively. We caught 10,066 fishes, weighting 128,486 g, distributed in 37 species belonging to 18 families that are similar in species composition to the dominant fish fauna Trachinotus marginatus (Cuvier, 1832), Atherinella brasiliensis (Quoy \& Gaimard, 1825), Brevoortia pectinata (Jenyns, 1842), Mugil platanus (Gunther, 1880), Menticirrhus americanus (Linnaeus, 1758), Menticirrhus littoralis (Holbrook, 1847), and Oncopterus darwinii (Steindachner, 1874) - usually collected in the beaches of Rio Grande do Sul with a smaller net. The dominant size classes were between 50 and $100 \mathrm{~mm}$ TL and represent $43 \%$ of total catch. Based on CCA analysis we suggested that the ichthyofauna is primarily structured by seasonal variation in the physicochemical and environmental parameters and secondarily by the spatial difference between areas nearer (< approximately $10 \mathrm{~km}$ ) or more distant (> approximately $10 \mathrm{~km}$ ) from the Patos Lagoon's jetties. The composition and dominance of the fish community of the surf zone of Cassino Beach changed little in the latest 20 years, indicating a possible stability of the system.
\end{abstract}

KEY WORDS. Beach seine; fish community; dominant species; multivaried analysis; wave exposition.

A zona de arrebentação de praias arenosas é um ambiente altamente dinâmico que abriga uma fauna de peixes caracterizada pela baixa diversidade e alta dominância de poucas espécies (Godefroid et al. 2003, Monteiro-Neto et al. 2003, Félix et al. 2007). Muitos estudos enfatizaram a importância dessas áreas como berçário de peixes juvenis de diversas espécies marinhas e estuarinas (LASIAK 1986, Bennet 1989, Monteiro-Neto \& Musick 1994, Gaelzer \& ZaLmon 2003).

A importância dos peixes juvenis nestes ambientes foi bem documentada em várias praias do litoral brasileiro por Gaelzer \& Zalmon (2003), Godefroid et al. (2003) e Vasconcellos et al. (2007). Padrão semelhante foi descrito para a Praia do Cassino, litoral do Rio Grande do Sul, por Monteiro-Neto et al. (2003), embora as redes de arrasto de praia, por eles empregadas, tivessem malhas menores e fossem operadas em áreas rasas, com profundidades inferiores a $1 \mathrm{~m}$, o que as tornavam seletivas para a ictiofauna juvenil. Neste caso, são inadequadas as inferências ecológicas derivadas de estudos da porção praial mais rasa e generalizadas para toda a zona de arrebentação.

Este trabalho teve como objetivo caracterizar as variações espaciais e sazonais da comunidade de peixes da Praia do Cassino, Rio Grande do Sul, em profundidades superiores a 1 $\mathrm{m}$, utilizando uma rede desenvolvida para profundidades de até 1,6 m e projetada para a captura tanto de peixes juvenis quanto de subadultos e adultos.

\section{MATERIAL E MÉTODOS}

A Praia do Cassino está localizada no litoral sul do Rio Grande do Sul e é caracterizada por uma grande faixa arenosa com aproximadamente $220 \mathrm{~km}$ de extensão. Ao longo desta praia, os estágios morfodinâmicos variam entre dissipativo e reflexivo e são influenciados sazonalmente pela ação de fortes ventos e ondulações que chegam pelo sul e sudeste do Oceano 
Atlântico, os quais afetam drasticamente a dinâmica da zona de arrebentação (CALLIARI et al. 2001).

As amostragens foram realizadas em três pontos da praia, o primeiro próximo ao Molhe Oeste da Barra da Lagoa dos Patos (1), o segundo localizado cerca de $6 \mathrm{~km}$ ao sul, em frente à Passarela da Praia do Cassino (2), e o último localizado a cerca de $10 \mathrm{~km}$ ao sul dos molhes, nas proximidades da Estação Marinha de Aquicultura da FURG - EMA (3). As coletas foram realizadas mensalmente durante o período da manhã, de outubro de 2005 a setembro de 2006 (Fig. 1).

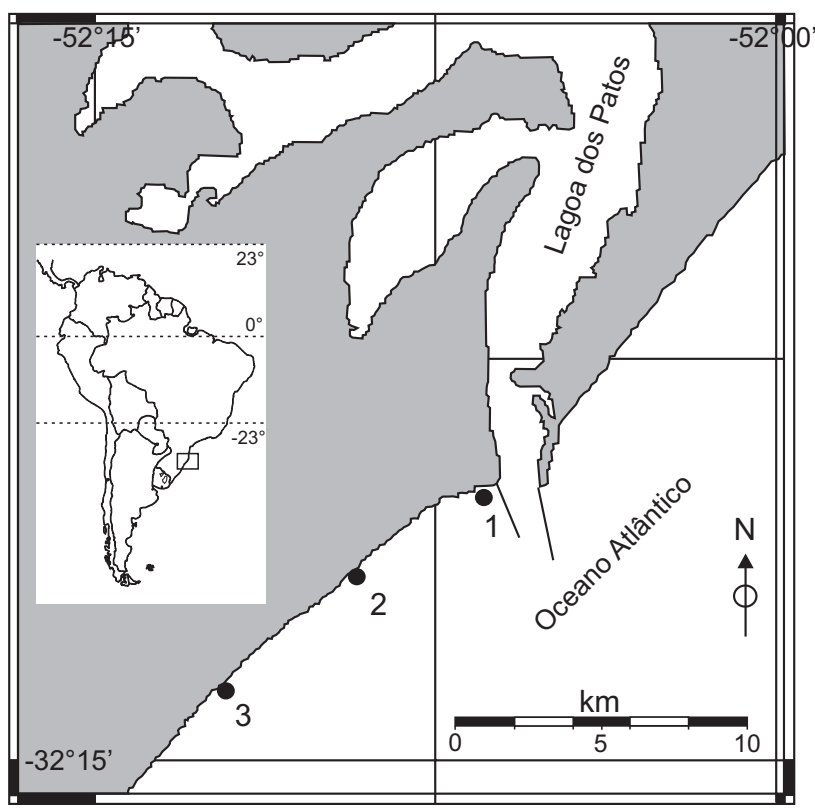

Figura 1. Mapa da área de estudo com detalhe da Praia do Cassino e dos locais de coleta.

A coleta dos dados foi feita com uma rede de arrasto de praia com $30 \mathrm{~m}$ de comprimento por 1,8 $\mathrm{m}$ de altura, tracionada por dois calões, um em cada extremidade. A rede foi confeccionada com nylon monofilamento em malha uniforme de $12 \mathrm{~mm}$ (entre nós opostos). Ao longo de toda a porção inferior da rede, entre o pano principal e a tralha do chumbo, há uma barra de $30 \mathrm{~cm}$ confeccionada de fio multifilamento com malha de 12 $\mathrm{mm}$. O objetivo da barra é evitar que a captura de siris Callinectes spp. danifique a rede. A malha multifilamento provoca um aumento do atrito do fio com a água e consequentemente um aumento da turbulência da água dentro da rede, reduzindo assim o escape de exemplares de pequeno porte $(<20 \mathrm{~mm})$, que geralmente escapariam pela malha $12 \mathrm{~mm}$ monofilamento.

Em cada ponto de coleta foram realizados cinco arrastos perpendiculares à praia, até a profundidade de 1,6 m. Os arrastos foram feitos no sentido da corrente e a área varrida pela rede, em cada arrasto, foi obtida através da distância perpendicular à praia no início do arrasto (Dper) e a distância paralela (Dpar), que corresponde ao deslocamento que a corrente de deriva provoca na rede ao longo do arrasto. A trajetória estimada da rede (Tr) foi estimada pela fórmula: $\operatorname{Tr}^{2}=\left(D p e r^{2}+D p a r^{2}\right)$, onde Tr é o equivalente à hipotenusa de um triângulo reto, e os lados do triângulo equivalem a Dper e Dpar. A abertura média da rede ( $\mathrm{A}$ = distância entre os dois calões) foi estimada em $20 \mathrm{~m}$ e o produto de Tr por A resulta em uma estimativa mais precisa da área varrida em cada arrasto (VIEIRA et al. 2006).

Para identificar as variáveis ambientais que mais influenciaram a comunidade de peixes da Praia do Cassino, foram registradas, em cada local amostrado, a temperatura superficial da água, salinidade, transparência da água, velocidade e direção do vento. Foi estimado o grau de exposição às ondas, pela observação da altura significativa de onda, número de bancos e cavas, na formação da topografia do fundo arenoso. Por fim, foi registrado o número de linhas de arrebentação, desde a primeira arrebentação (a mais forte) até a última próxima da praia, desconsiderando a área de varrido.

As amostras foram fixadas em formaldeído a $10 \%$ com água do local e etiquetadas. No laboratório, os peixes foram identificados até o nível de espécie segundo Figueiredo \& Menezes (1978, 1980, 2000), Menezes \& Figueiredo $(1980,1985)$ e Fischer et al. (2004). Foram medidos os comprimentos totais (CT) de no máximo 100 exemplares de cada espécie por amostra, sendo os restantes apenas contados. Por último, todos os indivíduos da mesma espécie e amostra foram pesados juntos.

Com base nos dados brutos de captura, número total de indivíduos por espécie, foi calculada a CPUAn (Captura numérica por unidade de Área varrida), definida como a razão entre o número de indivíduos capturados em cada arrasto e a área varrida pela rede (VIEIRA et al. 2006). O mesmo processo de tratamento dos dados foi realizado para obtenção da biomassa, denominada de CPUAb (Captura em peso por unidade de área varrida). Os cálculos do CPUAn e CPUAb foram padronizados para $100 \mathrm{~m}^{2}$ e os valores foram transformados em $\log _{10}(\mathrm{x}+1)$.

Com base nos valores médios de CPUAn foi determinada a contribuição numérica percentual (PN\%) de cada espécie em relação ao total capturado de todas as espécies ao longo do período estudado, assim como a frequência de ocorrência (FO\%) de cada espécie no total das amostras. As espécies com valores de $\mathrm{PN} \%$ e $\mathrm{FO} \%$ superiores as suas respectivas médias foram consideradas frequentes e/ou abundantes no período.

Devido à forte influência sazonal na variabilidade dos dados, a análise espacial foi realizada em separado para cada estação do ano (Primavera de outubro a dezembro de 2005, Verão de janeiro a março de 2006, Outono de abril a junho de 2006 e Inverno de julho a setembro de 2006). Com isto, foi possível identificar com mais consistência as diferenças e tendências de variação na estrutura da comunidade de peixes.

Para avaliação da variabilidade espaço-temporal dos dados físico-químicos, foi usado o teste de Kruskal-Wallis. A significância das variações sazonais e espaciais dos dados bio- 
lógicos foi testada por uma ANOVA unifatorial, sempre que atendidos os pressupostos de análise (homocedasticidade, normalidade e relação entre a variância e a média dos dados). Quando algum dos pressupostos para análise de variância não era atendido, foi aplicado o teste não-paramétrico de Kruskal-Wallis.

Para avaliação de diferença entre as médias dos parâmetros biológicos no tempo e no espaço foi utilizado o teste a posteriori de Tukey. Todos os testes, paramétricos e não paramétricos, foram aplicados com uma precisão de 5\% de significância.

Por ultimo, foi aplicada uma Análise de Correlação Canônica (CCA) para descrever as tendências de variabilidade biológica em função da variabilidade ambiental (Pessanha \& Araújo 2003). Para aplicar esta análise, foram usados tanto os dados abióticos como os bióticos normalizados através da transformação pelo $\log _{10}(\mathrm{x}+1)$ e apenas os dados abióticos foram padronizados pela seguinte equação: $(x-\mu) / \sigma$, onde $\mu$ é a média da amostra e $\sigma$ o desvio padrão (Field et al.1982).

A estrutura em comprimento da ictiofauna estudada foi analisada através da distribuição da abundância (CPUAn) por classe de comprimento total (CT) das espécies, que corresponde a Captura por Unidade de Área por Classe de Comprimento (CPUA-CC) (Garcia \& Vieira 2001, Vieira et al. 2006). Com base na distribuição geral da CPUA-CC foram separadas quatro grandes classes de comprimento (0-50, 50-100, 100-150 e >150 mm); Para estas e para o total das capturas, foram calculados os índices de riqueza e equitatividade, baseados nos valores absolutos de captura, objetivando interpretar melhor os parâmetros da diversidade.

A diversidade foi calculada através do número esperado de espécies (índice de rarefação $(\mathrm{E}(\mathrm{S})$ ) e a equitatividade das espécies pelo índice de Evar. Estes dois componentes da diversidade foram interpretadas através de suas representações gráficas (GARCia \& Vieira 2001). Para tanto, foram construídos gráficos tendo no eixo das abscissas o valor de Evar e no das ordenadas o valor de $\mathrm{E}(\mathrm{S})$. Nos pontos de intersecção de $\mathrm{x}$ e $\mathrm{y}$ foram inseridos os valores de CPUAn, graficados como círculos proporcionais a sua ordem de grandeza. O objetivo dessa técnica é interpretar, de forma independente, os dois componentes da diversidade (riqueza e equitatividade) e associá-los aos valores de abundância relativa (CPUAn) da comunidade.

\section{RESULTADOS}

A temperatura, salinidade, transparência da água e número de linhas de arrebentações apresentaram variações temporais significativas $(\mathrm{p}<0,001)$. As variações na altura de ondas, número de bancos e cavas na areia mostraram que a área de estudo é espacialmente heterogênea ( $\mathrm{p}<0,001)$ (Tab. I).

Em janeiro foi observado um valor máximo de $26,5^{\circ} \mathrm{C}$ nas águas da Praia do Cassino e valores mínimos de $12^{\circ} \mathrm{C} \mathrm{em}$ novembro. A salinidade mais alta $(34,7)$ foi observada no mês de dezembro e a mais baixa em maio $(23,9)$. A transparência da água foi máxima em julho $(140 \mathrm{~cm})$ e mínima em março (15). $\mathrm{O}$ número de linhas de arrebentação foi máximo durante o verão e mínimo na primavera (Fig. 2).
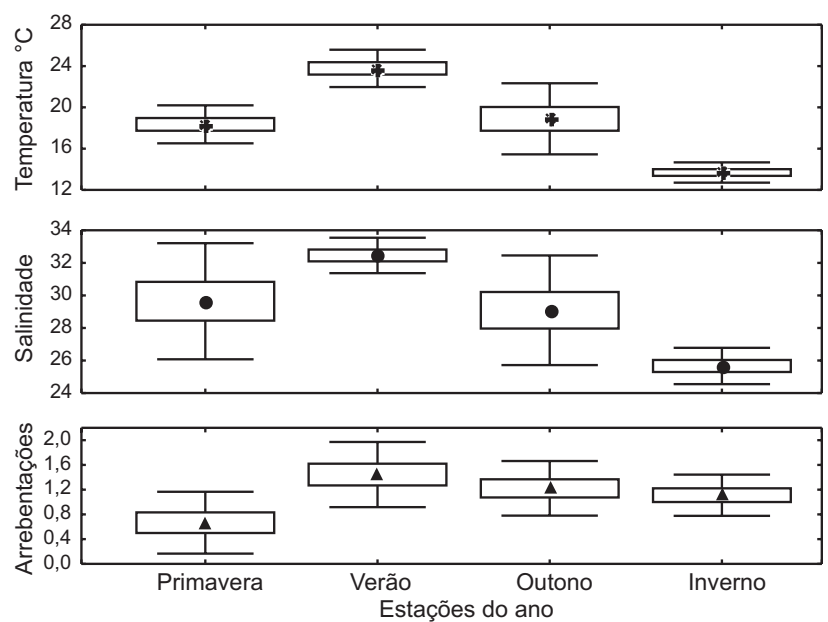

Figura 2. Variabilidade sazonal da temperatura, salinidade e número de linhas de arrebentação.

Tabela I. Sumário descritivo das variáveis físico-químicas que apresentaram diferenças espaço-temporal significativas.

\begin{tabular}{|c|c|c|c|c|c|c|c|c|c|c|}
\hline & \multirow{2}{*}{$\mathrm{N}$} & \multirow{2}{*}{ Média } & \multirow{2}{*}{ Mediana } & \multicolumn{2}{|c|}{ Intervalo de Confiança } & \multirow{2}{*}{ Minimo } & \multirow{2}{*}{ Máximo } & \multirow{2}{*}{ Desvio Padrão } & \multirow{2}{*}{$\begin{array}{l}\text { Erro Padrão } \\
\text { da média }\end{array}$} & \multirow{2}{*}{$\mathrm{p}$} \\
\hline & & & & $-95 \%$ & $+95 \%$ & & & & & \\
\hline \multicolumn{11}{|l|}{ Sazonal } \\
\hline Transparência & 36 & 45,83 & 40,00 & 35,02 & 56,64 & 15,00 & 140,00 & 31,95 & 5,32 & 0,0105 \\
\hline Temperatura & 36 & 18,68 & 17,50 & 17,26 & 20,10 & 13,00 & 26,50 & 4,19 & 0,70 & 0,0000 \\
\hline Salinidade & 36 & 29,21 & 27,90 & 28,04 & 30,39 & 23,90 & 34,70 & 3,47 & 0,58 & 0,0040 \\
\hline Arrebentação & 36 & 1,11 & 1,00 & 0,93 & 1,29 & 0 & 2,00 & 0,52 & 0,09 & 0,0165 \\
\hline \multicolumn{11}{|l|}{ Espacial } \\
\hline Altura de onda & 36 & 0,35 & 0,25 & 0,29 & 0,40 & 0 & 0,83 & 0,17 & 0,03 & 0,0042 \\
\hline Banco de areia & 36 & 0,61 & 1,00 & 0,43 & 0,80 & 0 & 2,00 & 0,55 & 0,09 & 0,0008 \\
\hline Cava na areia & 36 & 0,83 & 1,00 & 0,60 & 1,07 & 0 & 2,00 & 0,70 & 0,12 & 0,0013 \\
\hline
\end{tabular}


A praia do Cassino se diferencia espacialmente com o aumento da distância do molhe oeste. O ponto 1 (próximo aos molhes da barra) foi significativamente diferente dos pontos 2 e 3, apresentando menor altura de onda e com menos bancos e cavas na topografia do fundo arenoso (Fig. 3).
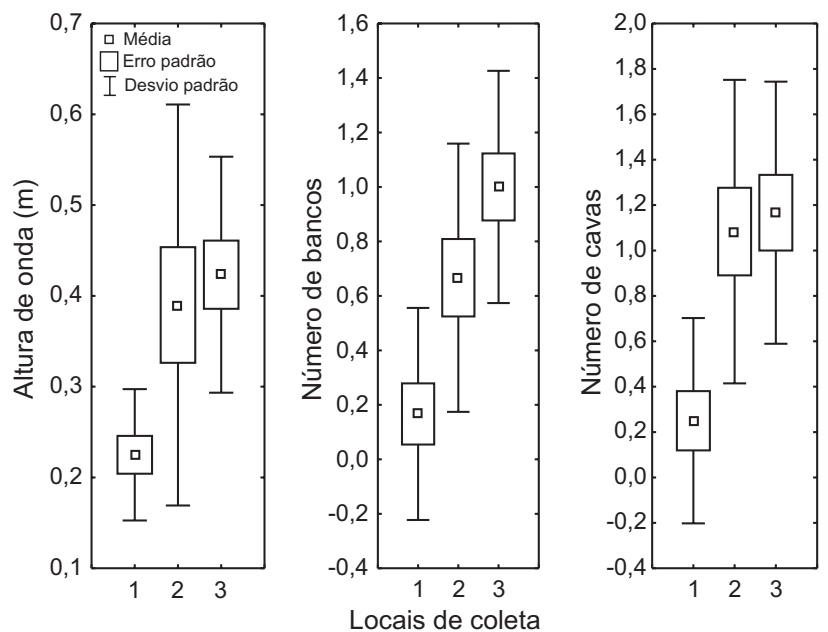

Figura 3. Variação da altura de onda, número de bancos e cavas por local de coleta.

Foi capturado nas 180 amostras de arrasto de praia um total de 10.066 indivíduos $(128,5 \mathrm{~kg})$, representados por 37 espécies pertencentes a 18 famílias. As famílias mais representativas em número de espécies (S) e número de indivíduos coletados (N) foram Carangidae $(\mathrm{S}=6, \mathrm{~N}=3343)$, Clupeidae $(\mathrm{S}=4, \mathrm{~N}=$ 2457 ) e Sciaenidae ( $\mathrm{S}=4, \mathrm{~N}=1402)$. Trachinotus marginatus (Cuvier, 1832) (33,1\%), Brevoortia pectinata (Jenyns, 1842) $(18,1 \%)$, Odontesthes argentinensis (Valenciennes, 1835) (14,6\%), Menticirrhus americanus (Linnaeus, 1758) (9,3\%), Menticirrhus littoralis (Holbrook, 1847) (8,8\%) e Mugil platanus (Gunther, 1880) $(3,5 \%)$ que perfizeram $87,4 \%$ da abundancia total de peixes capturados na zona de arrebentação da Praia do Cassino (Tab. II).

Das 37 espécies registradas, 20 apresentaram valores de CPUAn que correspondem a menos que $0,1 \%$ do total de peixes capturados. As demais 17 espécies, cujo somatório de CPUAn representou $99,3 \%$ do total de peixes capturados, foram selecionadas para as análises da estrutura de comunidade (Tab. II).

Dentre as 17 espécies mais abundantes e frequentes neste estudo, apenas 11 podem ser consideradas constantes na "surf zone" - T. marginatus, O. argentinensis, M. littoralis, B. pectinata, M. platanus, Pomatomus saltatrix (Linnaeus, 1766), Lycengraulis grossidens (Agassiz, 1829), Oncopterus darwinii (Steindachner, 1874), Mugil gaimardianus (Desmarest, 1831), Genidens barbus (Lacepède, 1803), Trichiurus lepturus (Linnaeus, 1758) -, enquanto que as demais seis espécies - M. americanus, Atherinella brasiliensis, Micropogonias furnieri (Desmarest, 1823), Sardinella brasiliensis (Eigenmann, 1894), Genidens planifrons (Higuchi, Reis \& Araújo, 1982) e Mugil curema (Valenciennes, 1836) - são aquelas que estão presentes na zona de arrebentação em somente uma determinada época do ano (Tab. II).

Os descritores da comunidade de peixes da Praia do Cassino (CPUAn, CPUAb e número de espécies) apresentaram interações significativas entre o espaço e o tempo (ANOVA Bifatorial $\mathrm{p}<0,05)$. Entretanto, esta interação acontece entre locais diferentes e em instantes diferentes, como pode ser observado na figura 4 e corroborado pelo teste de Turkey que mostrou, por exemplo, que a CPUAn no ponto 1 durante a primavera difere do ponto 3 no inverno. Portanto, foi aplicada uma ANOVA unifatorial para o espaço em cada estação do ano separadamente. O ponto 1 foi diferente $(\mathrm{p}<0,005)$ dos pontos 2 e 3 ao longo do ano para os parâmetros biológicos (Tab. III).

Durante os meses quentes, houve uma maior captura (CPUAn) nos pontos mais distantes do molhe (ponto 1). Porém nos meses frios, as maiores capturas ocorreram próximas ao molhe. Um padrão claro foi observado nas estações do outono e inverno, quando os valores dos parâmetros biológicos diminuíram com o aumento da distância do molhe (Fig. 4).

A correlação entre os parâmetros ambientais e biológicos, através da análise de correlação canônica (CCA), confirma os padrões espaciais e temporais já descritos anteriormente. Aproximadamente $41 \%$ da variabilidade dos dados biológicos foi explicada pelas oito variáveis ambientais, sendo a temperatura da água, número de linhas de arrebentação, altura de onda, salinidade e transparência da água as mais relevantes. Os dados biológicos foram altamente correlacionados, com o primeiro $(0,93)$ e com o segundo $(0,72)$ eixo (Tab. IV).

A sazonalidade climática tem uma forte influência na praia do Cassino, apresentando-se de forma homogênea em toda a área de estudo, mas condicionando uma distinta estrutura da comunidade de peixes em cada estação do ano (Figs 5 e 6).

A análise de CCA revela que ictiofauna da zona de arrebentação da praia do Cassino, desde o Molhe Oeste até $10 \mathrm{~km}$ ao sul, é estruturada sazonalmente pela temperatura da água e espacialmente pelo grau de exposição às ondas (altura de onda, número de linhas de arrebentação, bancos, cavas e transparência da água) (Figs 5 e 6 e Tab. IV).

As espécies B. pectinata, G. barbus, G. planifrons, T. marginatus, S. brasiliensis, M. littoralis, M. furnieri, M.curema e T. lepturus são positivamente correlacionadas com as temperaturas mais altas do verão e com a proximidade dos molhes da barra (ponto 1), determinando a preferência dessas espécies por águas mais calmas e quentes, (Figs 5-7). Entretanto, as espécies mais abundantes e frequentes ( $T$. marginatus e $B$. pectinata) também mostram uma preferência pelos pontos mais expostos a ondas (2 e 3) durante o outono e inverno. Outras espécies, como $A$. brasiliensis, O. argentinensis, O. darwinii, P. saltatrix, M. gaimardianus e $M$. platanus, ocorrem durante o inverno e primavera, principalmente no ponto 1 , porém, com expressiva frequência no 2 e 3, também (Figs 5-7). 
Tabela II. Lista das espécies por estações do ano e locais de coleta (1, 2 e 3) em ordem decrescente de abundancia relativa e freqüência de ocorrência, com comprimentos (CT) máximo, médio e mínimo. As espécies mais representativas estão representadas em escala de importância nas cores dos retângulos, do cinza claro ao preto. Seus valores estão em unidades de CPUAn (ind/100 m²).

\begin{tabular}{|c|c|c|c|c|c|c|c|c|c|c|c|c|c|c|c|}
\hline \multirow{2}{*}{ Espécies } & \multirow{2}{*}{ Código } & \multicolumn{3}{|c|}{ Primavera } & \multicolumn{3}{|c|}{ Verão } & \multicolumn{3}{|c|}{ Outono } & \multicolumn{3}{|c|}{ Inverno } & - Máx & \multirow{2}{*}{ Mín } \\
\hline & & 1 & 2 & 3 & 1 & 2 & 3 & 1 & 2 & 3 & 1 & 2 & 3 & Méd & \\
\hline T. marginatus & TRAMAR & 2,00 & 39,50 & 15,38 & 40,15 & 124,50 & 199,50 & 20,00 & 32,14 & 6,82 & 16,43 & 26,10 & 3,21 & 16376,7 & 20 \\
\hline B. pectinata & BREPEC & 2,33 & 6,25 & 4,88 & 59,73 & 25,25 & 107,75 & 71,54 & 2,42 & 3,66 & 3,03 & 0,21 & 0,16 & 19569,6 & 25 \\
\hline O. argentinensis & ODOARG & 72,67 & 56,67 & 55,63 & 1,49 & 2,25 & 0,25 & 2,49 & 4,26 & 3,57 & 1,85 & 26,40 & 3,44 & 320162,0 & 25 \\
\hline M. americanus & MENAME & 81,00 & 12,17 & 30,75 & 17,17 & 2,83 & 3,50 & 0,11 & & & & & & 378119,1 & 24 \\
\hline M. littoralis & MENLIT & 1,50 & 16,88 & 20,63 & 17,83 & 34,33 & 40,25 & 1,79 & 1,94 & 1,89 & 0,38 & 1,44 & 0,53 & 223105,8 & 23 \\
\hline M. platanus & MUGPLA & 5,17 & 12,08 & 10,00 & 1,98 & 11,92 & 2,25 & 0,50 & 4,20 & 0,57 & 6,91 & 0,37 & 0,10 & 44369,7 & 21 \\
\hline O. darwinii & ONCDAR & 7,67 & 7,17 & 20,50 & 0,33 & 0,25 & 3,75 & & & 0,08 & 0,12 & 0,41 & 0,46 & 13271,6 & 36 \\
\hline G. planifrons & GENPLA & & 0,17 & & 0,50 & 1,25 & 28,50 & & & & & & & 165121,6 & 104 \\
\hline M. gaimardianus & MUGGAI & 4,00 & 1,25 & 4,63 & & 0,17 & 6,00 & 10,78 & 0,10 & 0,10 & 0,64 & 0,07 & & 36077,6 & 31 \\
\hline M. curema & MUGCUR & & 0,13 & & 1,92 & 10,50 & 12,25 & 0,70 & & & & & & 22843,4 & 25 \\
\hline L. grossidens & LYCGRO & 0,67 & 0,67 & 0,13 & 1,08 & 5,58 & 3,25 & 0,63 & 2,60 & 4,23 & 0,06 & & 0,10 & 188138,8 & 64 \\
\hline P. saltatrix & POMSAL & 1,50 & 2,46 & 2,63 & 0,50 & 0,25 & 0,50 & 1,10 & 0,17 & 1,24 & 0,25 & 0,33 & 0,70 & 280132,8 & 81 \\
\hline M. furnieri & MICFUR & & & & 9,57 & 0,25 & 0,50 & & 0,10 & & & 0,33 & & 226150,4 & 80 \\
\hline S. brasiliensis & SARBRA & & & & 5,83 & 0,50 & & 0,30 & & & & & & 197172,0 & 77 \\
\hline G. $b a r b a$ & GENBAR & 0,25 & 0,33 & & 1,42 & 0,50 & 3,00 & 0,11 & 0,08 & & 0,59 & & & 286114,0 & 84 \\
\hline A. brasiliensis & ATHBRA & 3,92 & 0,63 & 0,25 & & & & 0,39 & 0,46 & 0,07 & 0,43 & & & 221122,2 & 82 \\
\hline E. argenteus & EUCARG & 1,50 & 0,17 & & 0,17 & & & 0,05 & & & 0,91 & 0,40 & & 168101,5 & 77 \\
\hline T. lepturus & TRILEP & 0,25 & 0,17 & & 1,42 & 0,33 & 0,25 & 0,15 & & & 0,13 & & & 1000650,2 & 415 \\
\hline H. clupeola & HARCLU & & & & 0,25 & & & 0,95 & 0,67 & 0,21 & & & & 15594,5 & 86 \\
\hline S. vomer & SELVOM & & & & 0,67 & 0,50 & & 0,08 & & & & & & 12575,6 & 52 \\
\hline P. orbignyanus & PARORB & 0,33 & 0,17 & & & 0,25 & 0,25 & & & & 0,05 & & & 182145,8 & 70 \\
\hline E. gula & EUCGUL & 0,50 & 0,17 & & 0,17 & & & & & & & & & 135128,5 & 123 \\
\hline P. paru & PEPPAR & & & & 0,31 & 0,17 & & & 0,08 & & & & & 263201,8 & 170 \\
\hline S. hispidus & STEHIS & 0,17 & & & & & & & & & 0,08 & 0,10 & & 18199,5 & 57 \\
\hline O. saurus & OLISAU & & & & & & & 0,20 & & 0,08 & & & & 8867,2 & 54 \\
\hline T. carolinus & TRACAR & & & & & & & 0,05 & & & 0,13 & & 0,10 & 213145,8 & 97 \\
\hline A. sexspinosus & ASTSEX & & & & & & & 0,25 & & & & & & 11290,0 & 77 \\
\hline C. latus & CARLAT & & & & & & 0,25 & & & & & & & 6565,0 & 65 \\
\hline C. spilopterus & CITSPL & & 0,25 & & & & & & & & & & & 9292,0 & 92 \\
\hline E. melanopterus & EUCMEL & 0,17 & & & & & & & & & & & & 9797,0 & 97 \\
\hline H. unifasciatus & HYPUNI & 0,17 & & & & & & & & & & & & 270270,0 & 270 \\
\hline P. cromis & POGCRO & & & & 0,17 & & & & & & & & & 266266,0 & 266 \\
\hline P. oligodon & POLOLI & & 0,13 & & & & & & & & & & & 171171,0 & 171 \\
\hline A. saxatilis & ABUSAX & & & & & & & & 0,10 & & & & & 7878,0 & 78 \\
\hline C. ruber & CARRUB & & & & & & & 0,10 & & & & & & 9386,5 & 80 \\
\hline O. oglinum & OPHOGLI & & & & & & & & 0,08 & & & & & 9898,0 & 98 \\
\hline A. marinii & ANCMAR & & & & & & & & & & & 0,08 & & 7373,0 & 73 \\
\hline Abundantes e fre & equentes & 185,8 & 157,4 & 165,4 & 162,6 & 221,6 & 412,0 & 112,3 & 49,4 & 22,5 & 32,0 & 56,2 & 8,8 & CPUAn & \\
\hline Abundantes & & 11,7 & 9,9 & 10,4 & 10,3 & 14,0 & 26,0 & 7,1 & 3,1 & 1,4 & 2,0 & 3,5 & 0,6 & $\% \mathrm{n}$ & \\
\hline Frequentes & & 19 & 20 & 11 & 21 & 19 & 17 & 21 & 15 & 12 & 16 & 12 & 9 & Número esp & pécies \\
\hline
\end{tabular}



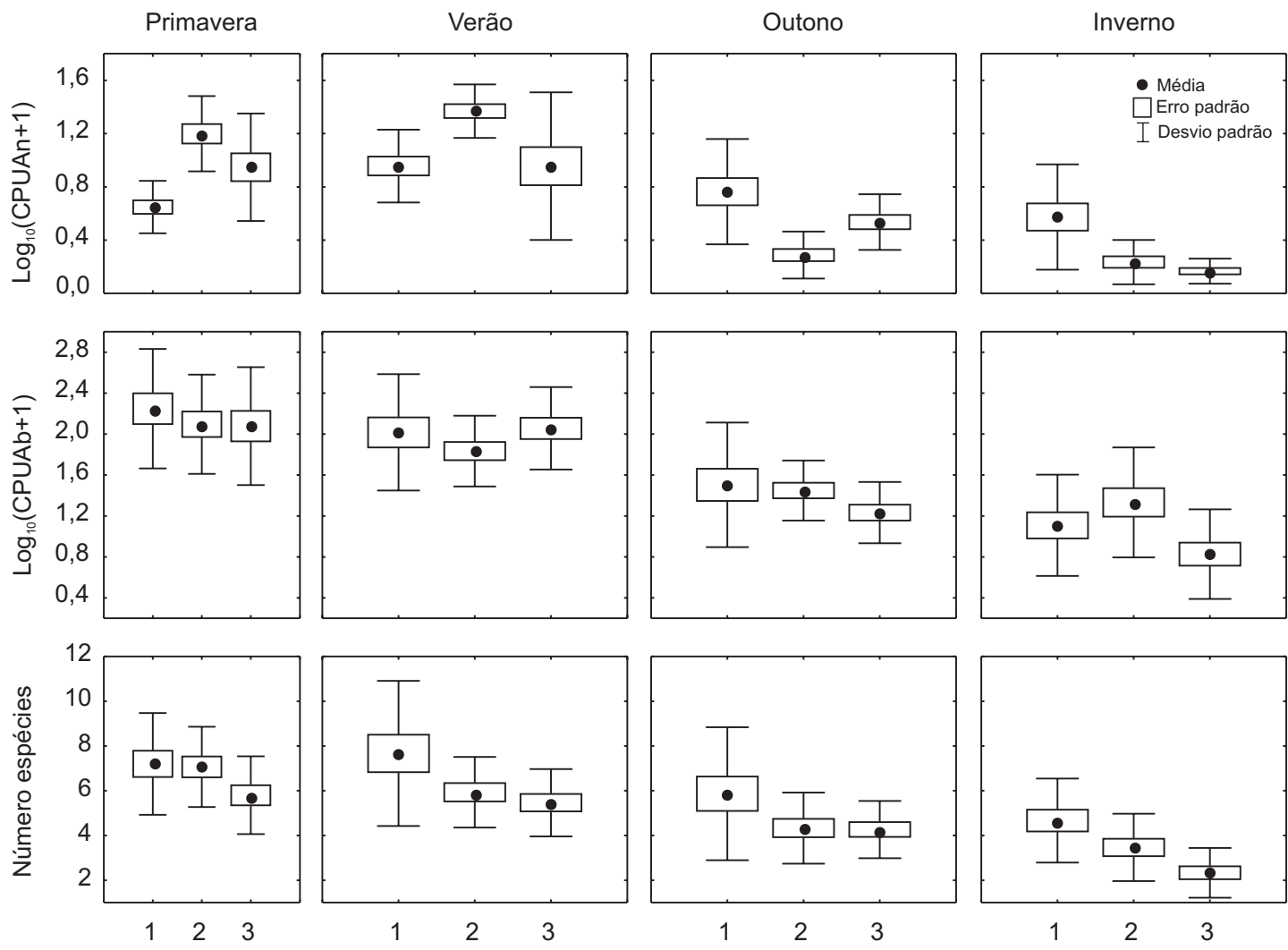

Figura 4. Variação espaço/temporal da CPUAn, CPUAb e número de espécies mostrados em função dos locais de coleta 1, 2 e 3.

Tabela III. Sumário dos dados bióticos e avaliação das diferenças espaciais e temporais através dos Testes da variância, Kruskal Wallis (K.W.) e Tukey. Todos os testes foram calculados com nível de significância de $5 \%$.

\begin{tabular}{|c|c|c|c|c|c|c|}
\hline Sazonal/Espacial & Média & Desvio Padrão & Erro Padrão da média & ANOVA (p) & K.W. (p) & Tukey (p)/Local \\
\hline CPUAn & 2,16 & 5,31 & 0,79 & 0,356 & 0,000 & $1=2=3$ \\
\hline Primavera & 2,14 & 0,87 & 0,13 & 0,001 & 0,000 & $1 \neq(2=3)$ \\
\hline Verão & 2,52 & 0,96 & 0,14 & 0,005 & 0,003 & $3 \neq 2 \neq 1=3$ \\
\hline Outono & 1,22 & 0,77 & 0,12 & 0,000 & 0,000 & $3 \neq 1=2 \neq 3$ \\
\hline Inverno & 0,75 & 0,71 & 0,11 & 0,000 & 0,000 & $1 \neq(2 \neq 3)$ \\
\hline CPUAb & 2,51 & 1,21 & 0,18 & 0,321 & 0,281 & $1=2=3$ \\
\hline Primavera & 4,93 & 1,25 & 0,19 & 0,651 & 0,656 & $1=2=3$ \\
\hline Verão & 4,53 & 1,04 & 0,15 & 0,361 & - & $1=2=3$ \\
\hline Outono & 3,21 & 1,00 & 0,15 & 0,195 & 0,116 & $1=2=3$ \\
\hline Inverno & 2,51 & 1,21 & 0,18 & 0,195 & 0,049 & $3=1=2 \neq 3$ \\
\hline Número de espécies & 5,34 & 2,45 & 0,18 & 0,000 & 0,072 & $1 \neq(2=3)$ \\
\hline Primavera & 6,69 & 2,01 & 0,30 & 0,107 & 0,121 & $1=2=3$ \\
\hline Verão & 6,36 & 2,40 & 0,36 & 0,027 & 0,129 & $1=2=3$ e $1 \neq 3$ \\
\hline Outono & 4,82 & 2,17 & 0,32 & 0,070 & 0,096 & $1=2=3$ \\
\hline Inverno & 3,49 & 1,78 & 0,27 & 0,001 & 0,001 & $3 \neq 1=2=3$ \\
\hline
\end{tabular}


Tabela IV. Sumário da Análise de Correlação Canônica (CCA) sobre a composição de espécies e respectivas abundâncias (CPUAn).

\begin{tabular}{|c|c|c|c|c|c|}
\hline \multirow{2}{*}{ Correlação das variáveis com os eixos } & \multicolumn{4}{|c|}{ Eixos } & \multirow{2}{*}{$\mathrm{p}<0,05$} \\
\hline & 1 & 2 & 3 & 4 & \\
\hline Temperatura da água & $-0,795$ & $-0,154$ & 0,053 & $-0,113$ & * \\
\hline Número de linhas de arrebentação & $-0,648$ & 0,381 & $-0,051$ & 0,033 & * \\
\hline Salinidade & $-0,517$ & $-0,022$ & 0,366 & $-0,008$ & * \\
\hline Altura significativa de onda & $-0,173$ & 0,509 & $-0,303$ & $-0,285$ & * \\
\hline Velocidade do vento & $-0,273$ & 0,123 & 0,298 & $-0,354$ & \\
\hline Número de cavas & $-0,036$ & 0,437 & 0,131 & $-0,248$ & \\
\hline Transparência da água & 0,340 & 0,270 & $-0,135$ & 0,399 & \\
\hline Número de bancos de areia & $-0,078$ & 0,368 & 0,110 & $-0,276$ & \\
\hline \multicolumn{6}{|l|}{ Resumo estatístico dos eixos } \\
\hline Autovalores & 0,238 & 0,101 & 0,081 & 0,041 & \\
\hline Correlações espécies - ambiente & 0,931 & 0,722 & 0,754 & 0,697 & \\
\hline \multicolumn{6}{|l|}{ Porcentagem de variância explicada } \\
\hline nos dados de espécies & 19,000 & 27,100 & 33,600 & 36,900 & \\
\hline na relação espécie - ambiente & 46,200 & 65,800 & 81,600 & 89,500 & \\
\hline Soma de todos os autovalores naturais & & & & & 1,249 \\
\hline Soma de todos os autovalores canônicos & & & & & 0,514 \\
\hline
\end{tabular}

* Indica a diferença significativa dos testes paramétricos e não-paramétrico.

A análise da abundância relativa por classe de tamanho (CPUA-CC) mostrou que os peixes com comprimentos totais (CT) menores que $50 \mathrm{~mm}$ corresponderam a $24 \%$ do total capturado, sendo representados pelas espécies $B$. pectinata, T. marginatus, $M$. platanus, M. curema e O. darwinii. Os peixes entre 50 e $100 \mathrm{~mm}$ CT foram os mais abundantes, correspondendo a $43,4 \%$ do total de peixes capturados, com aproximadamente $95 \%$ pertencendo às espécies T. marginatus, $B$. pectinata, M. littoralis, M. americanus e $O$. darwinii. Os indivíduos entre 100 e $150 \mathrm{~mm}$ CTs corresponderam a $21 \%$ da abundância total, e estavam representados, principalmente, por O. argentinensis, M. americanus, M. littoralis, G. planifrons, T. marginatus, B. pectinata, L. grossidens, M. furnieri e M.gaimardianus (Fig. 8). Os indivíduos maiores que $150 \mathrm{~mm}$ somaram 11,5\% da abundância total, e dentre estes as espécies O. argentinensis, $M$. littoralis, M. platanus, S. brasiliensis, M. americanus, P. saltatrix, $M$. furnieri e T. lepturus somaram mais de $95 \%$ dos peixes nesta classe de comprimento, sendo que T. lepturus teve representantes com até 1000 mm CT (Fig. 8 e Tab. II). Duas espécies (O. argentinensis e M. americanus) destacam-se por apresentarem aproximadamente $94 \%$ e $61 \%$ dos indivíduos capturados maiores que $100 \mathrm{~mm}$ CT, o que corresponde, respectivamente, a $42 \%$ e $17,6 \%$ de todos os indivíduos capturados acima de 100 mm CT (Fig. 8).

A estrutura em comprimento da ictiofauna variou significativamente ao longo do ano, com uma maior frequência de indivíduos pequenos ( $<100 \mathrm{~mm})$ durante o verão e os maiores durante a primavera (Fig. 8).
A diversidade da ictiofauna foi baixa, principalmente devido à baixa equitatividade ao longo de todo o ano. A figura 9 mostra a diversidade, através da visualização gráfica de seus dois componentes (riqueza e equitatividade) assim como a representação da abundância relativa (CPUAn) em escala proporcional de grandeza dos valores por estação do ano. Portanto, pode-se observar a menor diversidade de peixes para a classe de menor comprimento total $(50 \mathrm{~mm})$, com poucas espécies e uma baixa equitatividade. Por outro lado, foi observado um sensível aumento na diversidade para os indivíduos maiores que $150 \mathrm{~mm}$, principalmente devido ao aumento da equitatividade e abundância durante o verão.

\section{DISCUSSÃO}

A praia do Cassino, localizada no extremo sul do Brasil, é fortemente marcada pela variação sazonal nos parâmetros físico-químicos ambientais. Em geral, são observadas temperaturas e salinidades significantemente superiores no verão do que no inverno. Este fato influencia a estrutura de toda a biota que reside ou utiliza este ambiente em algum momento de seu ciclo de vida (Odebrecht et al. 1995, Busoli \& Muelbert 1999, Monteiro-Neto et al. 2003). Além disso, esta praia apresenta uma clara tendência de formação de um gradiente espacial na exposição a ondas, com o molhe oeste da barra da Lagoa dos Patos funcionando como uma barreira, durante a maior parte do ano, para as correntes e ondulações de nordeste e leste. Portanto, 

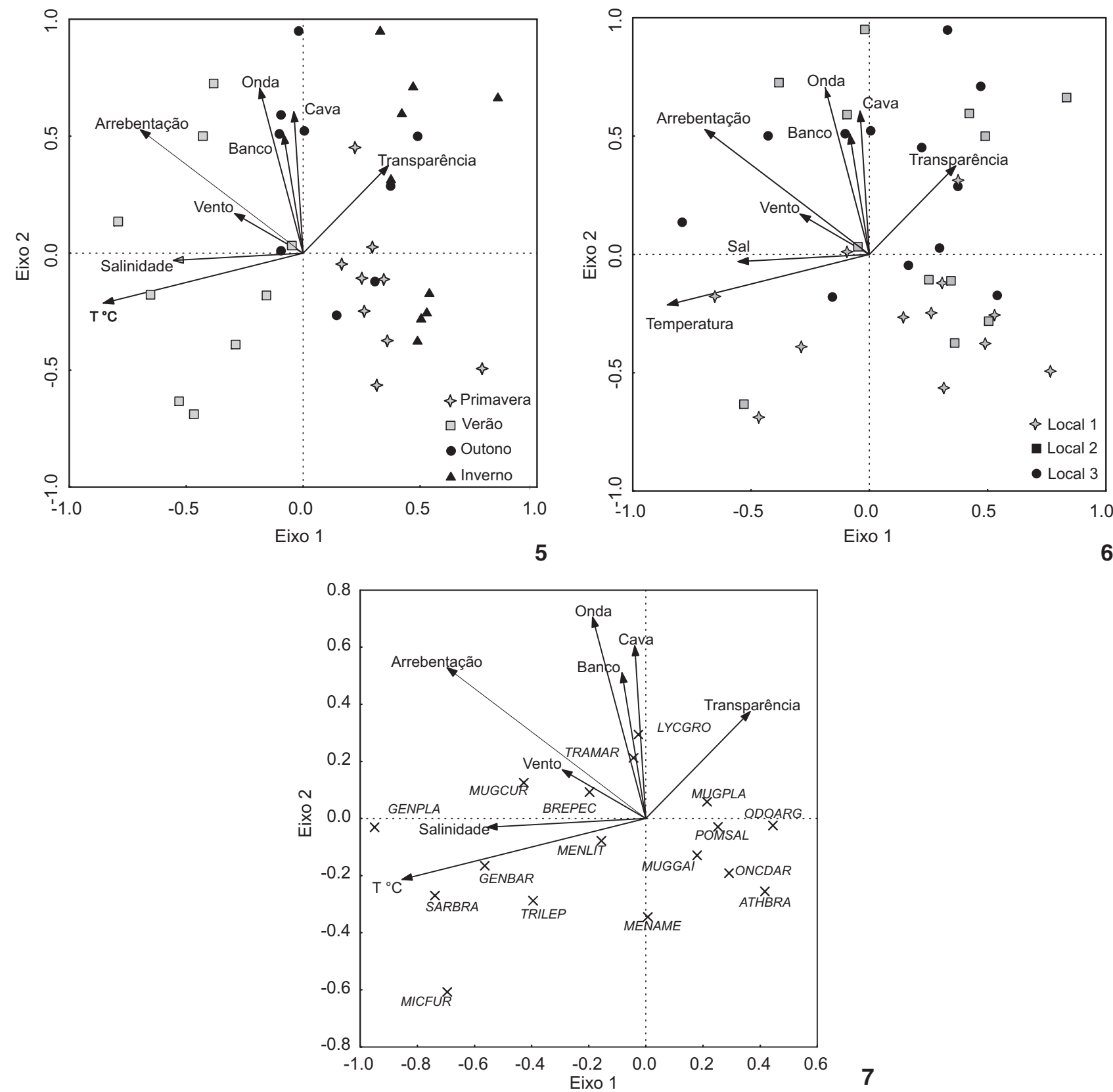

Figuras 5-7. Análise de Correlação Canônica (CCA) relacionando a abundância e composição de espécies com as variáveis ambientais: (5) representação sazonal das amostras (6) amostras espaciais,e (7) valores médios de abundância para cada espécies (ver código das espécies na tabela I). A variabilidade dos pontos (espécies, estações do ano e amostras espaciais) é explicada pelas variáveis ambientais que estão representadas pelos vetores.

com o aumento da distância desta estrutura artificial, há um aumento significativo na altura das ondas e na formação de bancos e cavas da topografia do fundo arenoso, assim como o aumento significativo no número de linhas de arrebentação na praia (Fig. 4), Em vista disso, os primeiros quilômetros dessa praia são caracterizados como uma área abrigada da ação das ondas e correntes de leste e nordeste (CAlliari et al. 2001).

Os molhes da Barra da Lagoa dos Patos funcionam também como a única via de conexão entre o estuário da Lagoa dos Patos e o oceano (Сhно et al. 1982), o que influencia a 

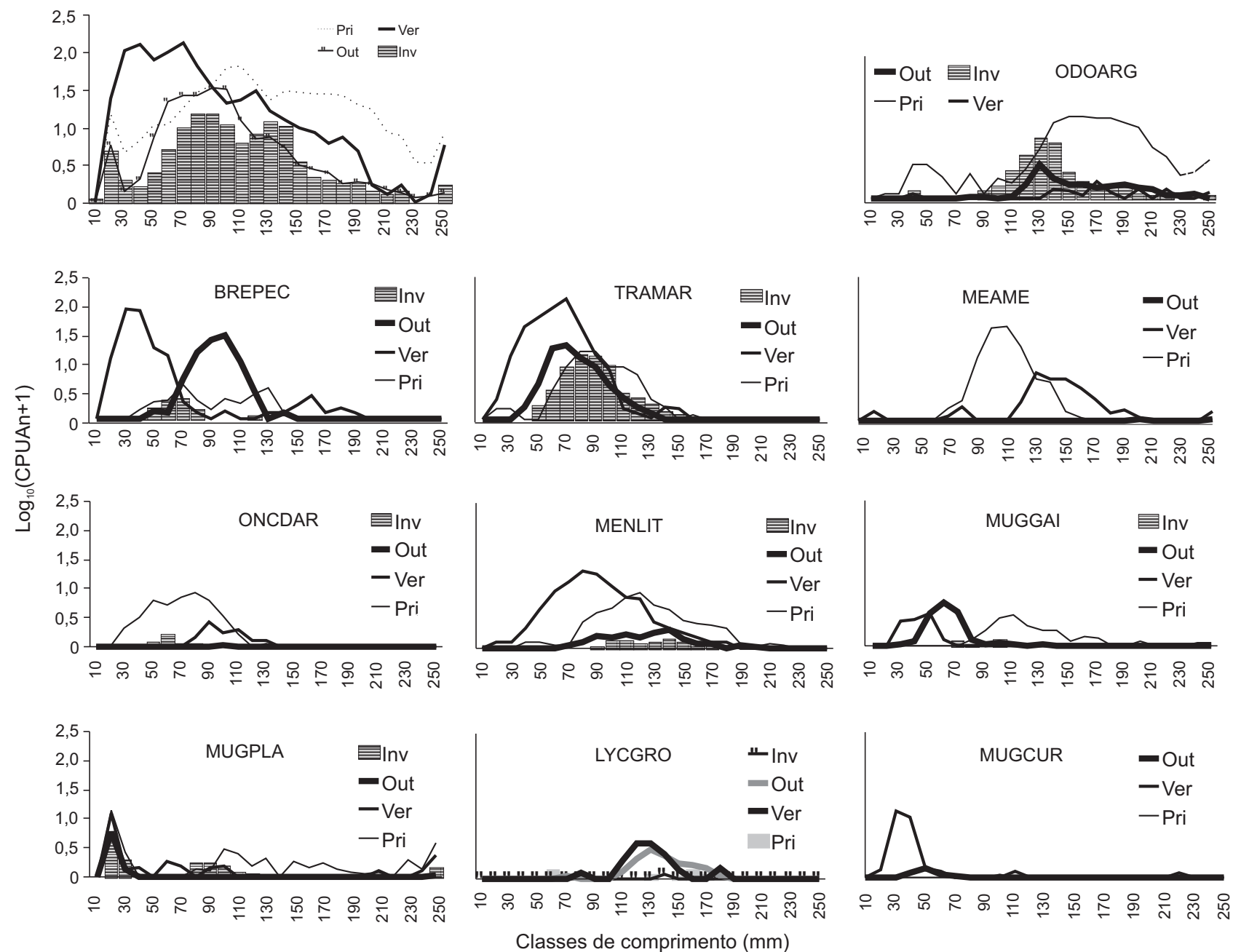

Figura 8. Distribuição geral da CPUA-CC e das 10 principais espécies por estação do ano (ver código das espécies na tabela I).

região costeira adjacente em determinadas épocas do ano, tornando-a uma extensão do estuário (Busoli \& Muelbert 1999), ou ainda uma via de acesso de espécies estuarino-relacionadas migrantes que recrutam no estuário da Lagoa dos Patos (Monteiro-Neto et al. 2003).

Como consequência dessa dinâmica, a ictiofauna apresenta-se claramente influenciada primeiramente pela forte sazonalidade da região sub-tropical e secundariamente pela presença dos molhes da barra da Lagoa dos Patos.

A equidade nas amostras foi baixa, mas observou-se uma tendência diretamente proporcional ao aumento do tamanho corporal, principalmente no verão, o que pode indicar uma tendência de mudança na estrutura da comunidade de peixes quando é possível capturar indivíduos maiores. Consequentemente percebe-se que a seletividade de uma rede de arrasto de praia pode ser determinante na avaliação da comunidade de peixes estudada (Monteiro-Neto \& Musick 1994, Jennings et al. 2001).
As dimensões $(30 \mathrm{~m})$ e o tamanho de malha $(12 \mathrm{~mm}) \mathrm{da}$ rede utilizada no presente estudo diferem da rede de $9 \mathrm{~m}$ e malha de 5 a $12 \mathrm{~mm}$, historicamente utilizada desde 1979 na região (Monteiro-Neto et al. 2003). A captura por estas redes convencionais seleciona peixes juvenis ou recém-recrutados que permanecem na zona de arrebentação durante alguns meses. Em seguida se deslocam para ambientes estuarinos onde encontram melhores condições para completar seu ciclo de vida ( Monteiro-Neto et al. 2003).

Apesar da semelhança entre as espécies capturadas neste estudo e aquelas registradas anteriormente, apenas 12 espécies (T. marginatus, A. brasiliensis, B. pectinata, G. barbus, L. grossidens, M. americanus, M. littoralis, M. curema, M. platanus, O. darwinii, Paralichthys orbignyanus (Valenciennes, 1842) e T. lepturus) foram comuns, sendo as mais abundantes e frequentes na praia do Cassino há mais de 25 anos. Contudo, Monteiro-Neto et al. (2003) observaram a presença de quatro espécies de água doce 

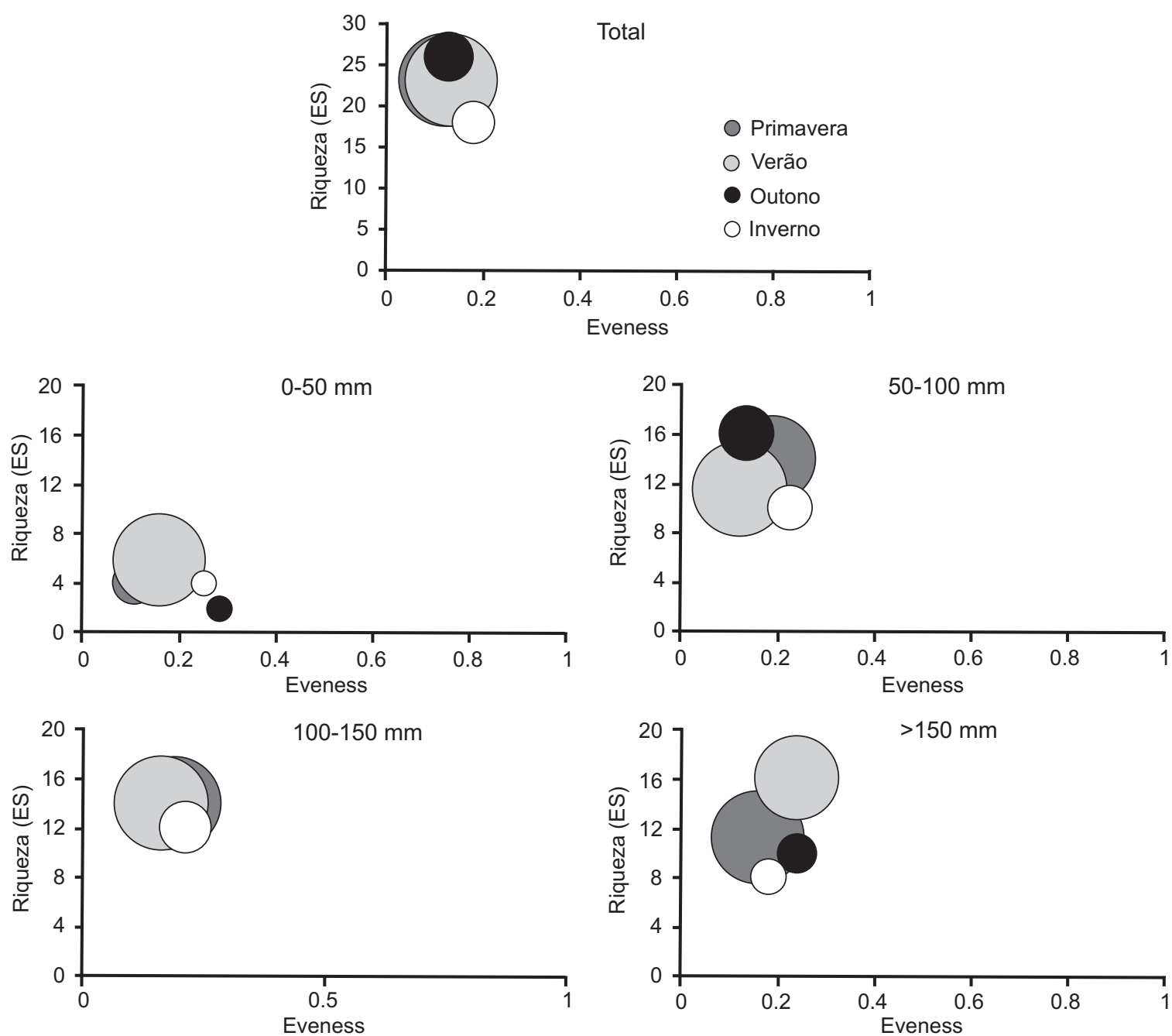

Figura 9. Diversidade geral da ictiofauna e por classe de comprimento. Riqueza de espécies (E(S)) no eixo y e equitatividade (Evar) no eixo $x$, o tamanho dos círculos são proporcionais aos valores da CPUAn para cada estação do ano: (P) primavera, (V) verão, (O) outono e (I) inverno.

- Characidium sp., Parapimelodus nigribarbis (Boulenger, 1889), Cheirodon interruptus (Jenyns, 1842) e Poecilia vivípara (Bloch \& Schneider, 1801) - e uma (Genidens genidens (Cuvier, 1829)) estuarino-residente, que não foram observadas no presente estudo. É possível, portanto, inferir que a composição das espécies, abundância e dominância permaneceram praticamente inalteradas ao longo desses anos, evidenciando a estabilidade da comunidade de peixes na praia do Cassino.

Por outro lado, o tamanho dos indivíduos coletados com a rede de $30 \mathrm{~m}$, neste estudo, foi superior àqueles capturados por Monteiro-Neto et al. (2003) que trabalharam na mesma praia, mas utilizaram a rede de $9 \mathrm{~m}$ descrita acima. Este padrão observado, também se diferencia do descrito por Clark et al. (1996a, b), Clark (1997), Godefroid et al. (2003) e Félix et al.
(2007) em outras praias do Brasil e do mundo. Nestes estudos citados, os peixes apresentavam um padrão de tamanho corporal inferior a $50 \mathrm{~mm}$ CT. Já no presente estudo, aproximadamente $75 \%$ dos peixes capturados eram maiores que $50 \mathrm{~mm}$ CT. Bennet (1989) usando uma rede de $25 \mathrm{~m}$ de comprimento por $2 \mathrm{~m}$ de altura com malha de $10 \mathrm{~mm}$ encontrou uma estrutura em comprimento para a comunidade de peixes de Fishoek "beach" diferente daquela observada em outros estudos. Segundo este autor há uma grande dificuldade em comparar comunidades de peixes de zona de arrebentação de diferentes localidades por causa das diferenças nas redes utilizadas neste tipo de estudo (tamanho de rede e malha), podendo em muitos casos subestimar a estrutura em comprimento da comunidade de peixes que habitam esse ambiente. 
A maior representatividade dos peixes maiores no presente estudo se deve ao tamanho da rede, tamanho de malha e ao fio monofilamento utilizado na confecção da rede de 30 m (MONTEIRONeto \& Musick 1994, Jennings et al. 2001). Uma rede confeccionada com fio monofilamento possui a vantagem de oferecer um menor atrito com a água, facilitando o manuseio da rede, tornando-a mais leve durante os arrastos, além de dificultar a percepção da rede pelos peixes. No entanto é mais frágil e requer manutenção mais frequente. Há limites para o tamanho e facilidade de manuseio da rede confeccionada com fio multifilamento devido ao peso durante o arrasto, demandando mais pessoas para manuseio. Contudo, tais comparações devem ser feitas com cautela, por abrangerem épocas, locais e amostradores diferentes. São necessários estudos comparativos mais detalhados dos amostradores e uma maior abrangência na captura de indivíduos maiores que $100 \mathrm{~mm}$. Porém, é clara a necessidade de se aprofundar mais o conhecimento da ictiofauna subadulta e adulta da zona de arrebentação externa, para que se possa melhor compreender a dinâmica desse ambiente como um todo.

Este estudo mostrou que apesar dos fatores climáticos influenciarem primariamente a praia do Cassino, os molhes da Barra da Lagoa dos Patos têm um importante papel na estruturação da comunidade de peixes, que se mostra estável há mais de 20 anos.

\section{AGRADECIMENTOS}

Ao CNPq pelo financiamento da bolsa de pesquisa e a toda a equipe do Laboratório de Ictiologia, Fundação Universidade do Rio Grande.

\section{LITERATURA CITADA}

BENNET, B.A. 1989. The fish community of a moderately exposed beach on the Southwestern Cape Coast of South Africa and an assessment of this habitat as a nursery for juvenile fish. Estuaries, Coastal and Shelf Science 28: 293-305.

Busoli, R.O. \& J.H. Muelbert. 1999. Composição taxonômica e distribuição do ictioplâncton na zona de arrebentação da Praia do Cassino $\left(32^{\circ} 10^{\prime} \mathrm{S}, 5^{\circ} 20^{\prime} \mathrm{W}\right)$. Atlântica 21: 19-35.

Clark, B.M. 1997. Variation in surf-zone fish community structure across a wave-exposure gradient. Estuarine, Coastal and Shelf Science 44: 659-674.

Clark, B.M.; B.A. Bennett \& S.J. Lamberth. 1996a. Temporal variations in surf zone fish assemblages from False Bay, South Africa. Marine Ecology Progress Series 131: 35-47.

Clark, B.M.; B.A. BennetT \& S.J. Lamberth. 1996b. Factors affecting spatial variability in seine net catches of fish in the surf zone of False Bay, South. Marine Ecology Progress Series 131: 17-34.

Chao, L.N.; L.E. Pereira; J.P. Vieira \& M. Bemvenuti . 1982. Relação preliminar dos peixes estuarinos e marinhos da lagoa dos patos e região costeira adjacente, Rio Grande do Sul, Brasil. Atlantica 1 (6): 67-75.
Calliari L. J.; N. Speranski; M. Torronteguy \& M.B. Oliveira. 2001. The mud banks of Cassino Beach, southern Brazil: characteristics, processes and effects. Journal of Coastal Research 34: 318-325.

Félix, F.C.; H.L. Spach ; O.S. Moro; J.R. Schwarz; C. Santos; C.W. Hackradt \& M.S. Hostim . 2007. Utilization patterns of surf zone inhabiting fish from beaches in Southern Brazil. PanAmerican Journal of Aquatic Sciences 2 (1): 27-39.

Field J. G.; K.R. Clarke \& R.M. WarWick. 1982. A Practical strategy for analysing multispecies distribution patterns. Marine Ecology Progress Series 8: 37-52.

Figueiredo, J.L. \& N.A. Menezes. 1978. Manual de peixes marinhos do sudeste do Brasil. II Teleostei (1). São Paulo, Museu de Zoologia, Universidade de São Paulo, 110p.

Figueiredo, J.L. \& N.A. Menezes. 1980. Manual de peixes marinhos do sudeste do Brasil. II Teleostei (3). São Paulo, Museu de Zoologia, Universidade de São Paulo, 110p.

Figueiredo J. L. \& N. A. Menezes. 2000. Manual de peixes marinhos do sudeste do Brasil. VI Teleostei (5). São Paulo, Museu de Zoologia, Universidade de São Paulo, 110p.

Fischer, L.G.; L.E.D. Pereira \& J.P. Vieira. 2004. Peixes estuarinos e costeiros. Rio Grande, Editora Ecoscientia, Série Biodiversidade do Atlântico Sudoeste, 127p.

GaELZER, L.R. \& I.R. ZALMON. 2003. The influence of wave gradient on the ichthyofauna of southeastern Brazil: Focusing the community structure in surf-zone. Journal of Coastal Research 35: 456-462.

Garcia, A.M. \& J.P. Vieira. 2001. O aumento da diversidade de peixes no estuário da Lagoa dos Patos durante o episódio El Niño 1997-1998. Atlântica 23: 133-152.

Godefroid, R.S.; H.L. Spach; R.J. Schwarz \& M.G. Queiroz. 2003. A fauna de peixes da praia do Balneário Atami, Paraná, Brasil. Atlântica 25 (2): 147-161.

Jennings, S.; M.J. Kaise \& J.D. Reynolds. 2001. Marine Fisheries Ecology. London, Backwell Science, 432p.

LASIAK, T.A. 1986. Juveniles, food, and the surf zone habitat: implications for the teleost nursery areas. South African Journal of Zoology 21: 51-55.

Menezes, N.A. \& J.L. Figueiredo. 1980. Manual de peixes marinhos do sudeste do Brasil. VI Teleostei (3). São Paulo, Museu de Zoologia, Universidade de São Paulo, 96p.

Menezes, N.A. \& J.L. Figueiredo. 1985. Manual de peixes marinhos do sudeste do Brasil. V Teleostei (4). São Paulo, Museu de Zoologia, Universidade de São Paulo, 105p.

Monteiro-Neto, C. \& J.A. Musick. 1994. Effects of beach seine size on the assessment of surf-zone fish communities. Atlantica 16: 23-29.

Monteiro-Neto, C.; L.P.R. Cunha \& J.A. Musick. 2003. Community structure of surf-zone fishes at Cassino Beach, Rio Grande do Sul, Brazil. Journal of Coastal Research 35: 492-501.

Odebrecht, C.A.Z. Segatto \& C.A. Freitas. 1995. Surf-zone chlorophyll $a$ variability at Cassino Beach, Southern Brazil. Estuaries, Coastal and Shelf Science 41: 81-90. 
Pessanha, A.L.M. \& F.G. Araújo. 2003. Spatial, temporal and diel variations of fish assemblages at two sandy beaches in the Sepetiba Bay, Rio de Janeiro, Brazil. Estuarine, Coastal and Shelf Science 57: 817-828.

Vasconcellos, R.M. \& J.N.S. Santos; M.A. Silva \& F.G. Araújo. 2007. Efeito do grau de exposição às ondas sobre a comuni-

Submitted: 14.X.2009; Accepted: 09.IX.2009.

Editorial responsibility: Paulo da Cunha Lana dade de peixes juvenis em praias arenosas no Município do Rio de Janeiro, Brasil. Biota Neotropical 7 (1): 93-100.

Vieira, J.P. ; T. GIarRIZzo ; H. SpaCh. 2006. Necton, p. 145-147. In: P.C. Lana; A. Bianchinl; C. Ribeiro; L.F.H. Niencheski; G. Filmann \& C.S.G. SANTOS (Eds). Avaliação ambiental de estuários brasileiros: diretrizes metodológicas. Rio de Janeiro, Museu Nacional, 155p. 